\title{
Clinical advances in the evaluation of patients with excessive central airway collapse: a narrative review
}

\author{
Karthika Ramakrishnan ${ }^{1}$, Maruti Kumaran ${ }^{1}$, Ryan Townsend ${ }^{2}$, Mark Weir ${ }^{2}$ \\ ${ }^{1}$ Department of Radiology, Lewis Katz School of Medicine at Temple University, Philadelphia, PA, USA; ${ }^{2}$ Department of Thoracic Medicine and \\ Surgery, Lewis Katz School of Medicine at Temple University, Philadelphia, PA, USA \\ Contributions: (I) Conception and design: None; (II) Administrative support: None; (III) Provision of study materials or patients: None; (IV) \\ Collection and assembly of data: None; (V) Data analysis and interpretation: None; (VI) Manuscript writing: All authors; (VII) Final approval of \\ manuscript: All authors. \\ Correspondence to: Mark Weir. Department of Thoracic Medicine and Surgery, Lewis Katz School of Medicine at Temple University, Philadelphia, \\ PA, USA. Email: mark.weir@tuhs.temple.edu.
}

\begin{abstract}
Excessive central airway collapse (ECAC) is an often under-recognized and misdiagnosed condition with significant symptom burden and attributable morbidity. This literature review focuses on the definition of ECAC and its spectrum of underlying conditions, associated co-morbidities, and primarily its clinical evaluation and diagnostic challenges in adults. ECAC is the umbrella term for large airway collapse, either from tracheobronchomalacia (TBM) — a condition characterized by loss of cartilaginous integrity, or from excessive dynamic airway collapse (EDAC) — due to laxity and collapse of the unsupported airway wall during increased pleural pressure. The symptoms, evaluation and interventions for both TBM and EDAC are similar. Patients will often complain of a "barking" cough, dyspnea on exertion, wheezing and recurrent infections. ECAC is most associated with obesity and chronic obstructive pulmonary disease (COPD). Clinical evaluation requires thorough assessment of respiratory symptoms and complaints, and for the presence of pulmonary and systemic comorbidities, pulmonary function test (PFT), dynamic CT (computed tomography) imaging studies and finally awake dynamic bronchoscopy. Dynamic CT studies and bronchoscopy that demonstrate $>50 \%$ airway collapse is considered diagnostic of ECAC. However, a $>50 \%$ collapse is not an uncommon finding among healthy individuals - thus differentiating what constitutes a pathologic collapse which warrants intervention is difficult. We recommend a multidisciplinary evaluation by teams familiar with the assessment and management of this under-recognized condition, while prioritizing optimization of medical management prior to surgical intervention.
\end{abstract}

Keywords: Excessive central airway collapse (ECAC); tracheobronchomalacia (TBM); excessive dynamic airway collapse (EDAC);

Received: 02 February 2021; Accepted: 12 August 2021; Published: 20 April 2022.

doi: 10.21037/jovs-21-6

View this article at: https://dx.doi.org/10.21037/jovs-21-6

The aim of this literature review is to clearly define excessive central airway collapse or ECAC, the underlying pathophysiology, discuss patient symptoms and comorbidities, relevant investigations and bronchoscopic evaluation. It is complimentary to a comprehensive series of review articles addressing all aspects of ECAC. Therapeutic bronchoscopy, stent trials and surgical management are outside the scope of this review. A search of PubMed and
Google scholar was conducted using the term 'Excessive central airway collapse', results were limited to the period 2000-2021, research studies, with full text availability and English language. We present the following article in accordance with the Narrative Review reporting checklist (available at https://jovs.amegroups.com/article/ view/10.21037/jovs-21-6/rc).

ECAC is considered when there is at least a $50 \%$ 

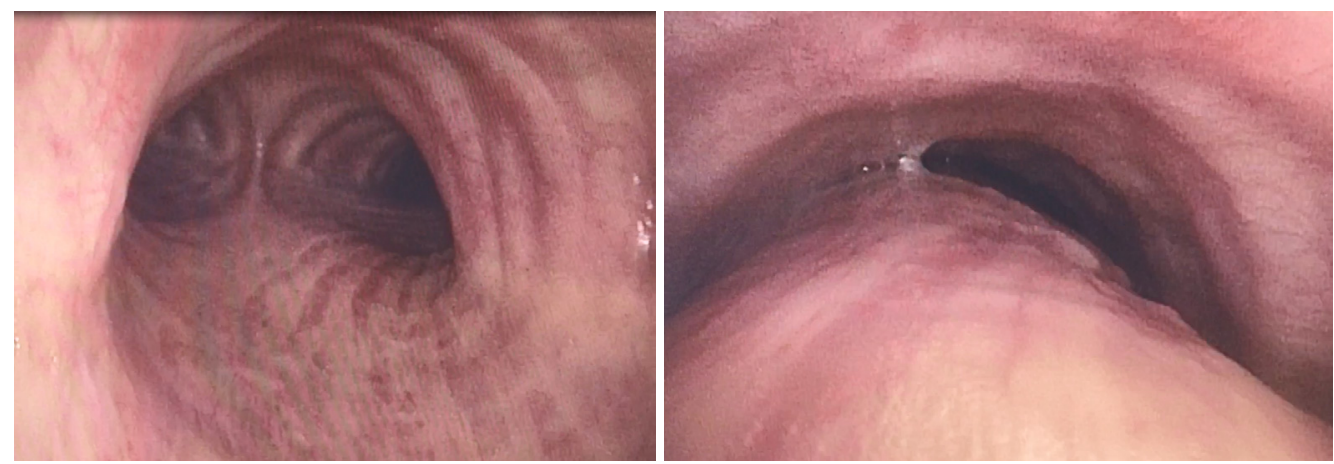

Figure 1 Bronchoscopy images showing paired inspiratory, expiratory breaths at the level of the distal trachea.

reduction in cross-sectional area of the central airways during expiration (1). However, there has been a wide range of tracheal collapsibility observed in healthy volunteer CT studies (2). The threshold at which ECAC is considered pathologic is a subject of debate. Most centers consider complete or near-complete collapse $(>90 \%$ reduction in cross-sectional area) significant enough to warrant consideration of intervention (3).

This reduction in cross sectional area can be caused by excessive dynamic airway collapse (EDAC) or tracheobronchomalacia (TBM). In EDAC, increased laxity of atrophic fibers in the tissues of the posterior membrane causes excessive invagination during exhalation (3) (see Figure 1). This defect is thought to result from interactions between pleural pressures, elastic recoil, airway compliance, and peripheral airway resistance (4).

TBM is defined as a loss of cartilaginous integrity of the trachea or bronchi. TBM can be present with or without dynamic invagination of the posterior membranous wall. TBM can present as a segmental/focal form or be diffuse, with global airway involvement. This diffuse form often progresses over time from an initial isolated segmental presentation (3). Focal TBM is often related to local injury from previous instrumentation (from endotracheal tube or tracheostomy) (5), radiotherapy, or pressure from vascular or malignant structures (6). Diffuse TBM can be seen in congenital cartilaginous disease (such as Mounier-Kuhn syndrome), relapsing polychondritis (7), chronic inflammatory lung conditions (such as emphysema), cystic fibrosis, and even exposure to toxins such as Mustard Gas (3).

However, for the practicing clinician, the importance is to recognize the presence of ECAC rather than the subtleties between TBM and EDAC-the symptoms, morbidity, medical management and interventions for both conditions are quite comparable (5).

\section{Physiology and anatomy}

The upper airway extends from the pharynx to the carina. The trachea begins below the Cricoid cartilage and extends to the main carina, approximately $10-16 \mathrm{~cm}$. The inner diameter is $21-27 \mathrm{~mm}$ and it is supported anteriorly and laterally by $15-20 \mathrm{C}$-shaped, cartilaginous rings. The anterior cartilaginous rings and the trachealis muscle of the posterior membranous wall aim to stabilize the airways against collapse during expiration.

Some degree of large airway collapse is normal. Dynamic CT studies of individuals with normal lung function have shown mean levels of expiratory collapse of $>50 \%$ in the trachea, the right main bronchus, the left main bronchus (LMB), and even total collapse in the bronchus intermedius (2).

There is a variable point in the intrathoracic airway where intraluminal and extraluminal pressures become equal. This is known as the equal pressure point (EPP); positive alveolar pressure (created by lung elastic recoil and the muscles of respiration) is the driving pressure that causes gas to flow through airways during expiration. This pressure $(\mathrm{Palv})$ is determined by the recoil pressure of the lungs (Pst) and the pleural pressure $(\mathrm{Ppl})$ :

$$
\text { Palv }=\text { Pst }+ \text { Ppl }
$$

A positive Palv creates a pressure gradient and flow of air from the alveoli toward the mouth. Due to the pressure gradient, the intraluminal pressure (Lateral pressure, PL) of the airway eventually becomes equal to pleural pressure $(\mathrm{Ppl})$. Where this occurs is the EPP, which divides the 
airways into upstream segments (toward alveoli from the EPP) - in which transmural pressure [PL-Ppl] is positive [i.e., intraluminal pressure $>$ pleural pressure, airway is patent), and downstream segments (toward the mouth from the EPP), in which the transmural pressure is negative within the intrathoracic airways (i.e., intraluminal pressure $<$ pleural pressure, airway is collapsed). This region of airway compression is referred to as a flow-limiting segment (FLS). Complete airway collapse will occur unless there is adequate cartilaginous support or traction provided by neighboring alveoli. This explains the tendency of the posterior membrane of the trachea and mainstem bronchi to collapse during expiration (8).

As lung volume decreases during expiration, elastic recoil pressure reduces. As a result, alveolar pressure is reduced and the EPP moves toward the alveoli. This usually results in the flow-limiting segment being in the lobar or segmental airways (9) toward residual volume. These lobar and segmental locations of FLS have been demonstrated in normal individuals and individuals with obstructive ventilatory impairment over considerable ranges of lung volume (10). The Starling resistor model shows that once the pressure drop occurs across the flow-limiting segment, proximal airway (downstream) resistance should not affect flow. Indeed, intraluminal pressure catheters demonstrate a lack of any further pressure drop in airways between the flow-limiting segment and the mouth (9).

The above principle explains how changes in lung compliance and airway resistance result in increased compression of the airway downstream from the FLS. For instance, a decrease in elastic recoil of the lungs (either because of low lung volume as seen in morbid obesity or because of emphysema) reduces the airway pressure relative to pleural pressure, resulting in greater dynamic compression. Further, chronic underlying lung pathology such as emphysema and chronic bronchitis may lead to long-standing excessive airway pressure gradients, which causes chronic atrophy and strain of longitudinal smooth muscle fibers resulting in increased wall compliance (11).

As for airway resistance, the greater the pressure gradient from the alveoli to the mouth, the sooner the development of an EPP/FLS and the greater the dynamic compression. However, this implies that central airway compression (bronchoscopic/radiographic EDAC) downstream from the flow-limiting segment (lobar/ segmental airways) is not pathologic from a flow dynamic standpoint (12). Consistent with this was a study of excessive expiratory tracheal collapse defined as $>80 \%$ expiratory reduction in tracheal luminal cross-sectional area during dynamic CT was shown to not significantly correlate with the pulmonary function tests (PFTs) or quality-of-life (QOL) measures (13).

\section{History and physical}

\section{Clinical features}

TBM/EDAC has been found to be present in up to $13 \%$ of all patients undergoing an evaluation for any respiratory complaint (14) and up to $23 \%$ of all patients evaluated for chronic bronchitis (15). Patients typically complain of shortness of breath, dyspnea on exertion (which commonly occurs when lifting or carrying heavy objects, laughing, or on forced exhalation-activities that create high intrapleural pressures), orthopnea, chronic cough (often described as "barking" in nature), inability to clear secretions, and recurrent infections/pneumonias. As per guidelines unexplained persistent cough should undergo a systematic protocol-based assessment (16). There is limited evidence to support the benefit of multidisciplinary cough clinics (17). Speech therapy evaluation as a part of a multidisciplinary clinic and a trial of non-pharmacological interventions such as speech therapy have been shown to be beneficial with no reported adverse events (18). The patient should undergo full physical exam paying close attention to the upper airway and assessing the effects of forced exhalation on auscultation and symptoms.

TBM/EDAC is often seen in patients with other common respiratory conditions such as asthma, chronic bronchitis, and emphysema, and even the inability to wean off of a ventilator. The myriad of overlapping symptoms often complicates the picture and can lead to misdiagnosis or poor response to medical therapy. Conversely, a study of 6 enlisted military individuals describes symptomatic TBM/EDAC found with no other medical co-morbidities, a very high exercise tolerance, and no history of any chronic lung disease. Their only presentation was the onset of dyspnea and wheezing on significant exercise and exertion (4).

Furthermore, ECAC is not always a static phenomenon-a follow up CT study of bronchiectasis patients previously found to have ECAC showed widespread variability with $45 \%$ improving, $20 \%$ unchanged and $35 \%$ progressing (19).

There is no ECAC-specific scale or questionnaire. A series 
of respiratory related standardized questionnaires have been used as research tools, such as the baseline functional status (Karnofsky Performance Scale); modified Medical Research Council (mMRC) dyspnea scale; respiratory related quality of life assessment (St. George Respiratory Questionnaire) and Cough Specific Quality of Life Questionnaire (CQLQ).

\section{Co-morbidities}

One of the most frequently identified co-morbidities with ECAC is obesity. In one study of 189 patients, $66.2 \%$ percent of patients with ECAC were classified as obese (BMI $>30 \mathrm{~kg} / \mathrm{m}^{2}$ ), and $35.2 \%$ had concurrent obstructive sleep apnea (OSA) (20). In another study, $50 \%$ of patients diagnosed with TBM/EDAC had a classification of morbid obesity (5). The degree of ECAC is worse in COPD patients with $\mathrm{BMI}>35$, and is associated with reduced exercise capacity and worse symptom burden (13). In a study looking at the relationship of obesity and asthma on central airway collapse, BMI was shown to be the strongest factor (21). Choo et al. suggests that anatomic changes associated with obesity include diaphragm elevation and the downward movement of the chest wall, which results in foreshortening and reduced rigidity of the trachea and increased pleural pressure (22).

Gastro-esophageal reflux disease (GERD) is thought to be a contributor to ECAC either as a causal or exacerbating factor. We know from the study of infant and child populations that GERD is highly related to the development of tracheomalacia and laryngomalacia, and is present in up to $70 \%$ of cases (20). Kandaswamy et al. suggests that repeated exposure of the airways to stomach acid and digestive enzymes alters the matrix protein structure of tracheal tissue resulting in reduced tensile strength of airway walls (23). A study by Castellanos et al. found 8 out of 10 patients with severe TBM to have significant GERD (5). In another study by Majid et al., $45 \%$ (63 of 139$)$ of patients with TBM $(33.3 \%)$ or EDAC $(51.1 \%)$ had GERD by abnormal esophageal $\mathrm{pH}$ testing. Additionally, obesity has been shown in multiple epidemiologic studies to be associated with GERD, the prevalence of which increases linearly with BMI. In those patients who also have concurrent OSA, their symptoms of GERD are likely worsened by the fact that during obstructive events, high negative intrathoracic pressures are generated distal to the pharynx and can worsen reflux events and aspiration (20).

Notably in the report by Majid et al., 29 of the 63 patients with GERD and TBM/EDAC self-reported improvement in their respiratory symptoms following treatment of their reflux (either medical or surgical). Because of these findings, we routinely evaluate for and treat GERD prior to any surgical intervention for ECAC. Additionally uncontrolled GERD can have a negative impact on surgical outcomes for tracheobronchoplasty (3) and those with refractory GERD being considered for surgical TBM intervention are often considered for a Nissen fundoplication or other concurrent surgical management of GERD (5).

\section{Investigations and testing}

In addition to diagnostic testing for TBM itself, it is important to evaluate co-morbidities that may contribute to poorly controlled symptoms. Evaluation of upper airway cough contributors such as chronic sinusitis, postnasal drip, allergies, routine assessment for gastroesophageal reflux symptoms which can be done by esophageal $\mathrm{pH}$ monitoring and EGD/endoscopy and optimize GERD treatment prior to intervention for ECAC. Due to the high prevalence of co-morbid sleep apnea we assess for symptoms of sleep apnea and perform routine sleep studies to evaluatethere should be a low threshold for testing and initiating treatment for OSA in the setting of suspected ECAC.

PFT and lung spirometry are routinely performed in the initial diagnostic workup, but even patients with moderate to severe ECAC can demonstrate normal flow-volume loops. In a study of 6 military individuals with TBM, all 6 had no evidence of intrathoracic or extrathoracic obstruction on their flow-volume loops (4). In a retrospective analysis of 90 patients with moderately to severely symptomatic TBM, Majid et al. found the presence of either an obstructive ventilatory defect, likely restrictive defect or mixed defect in $79 \%$ of the cases. However, the remaining $21 \%$ of patients had normal spirometry values, and $17 \%$ of available flow-volume loops were normal in appearance.

This same review highlighted several different loop qualities, which may suggest clinically important ECAC(Figure 2) notching of the expiratory curve (7 patients, 9.2\%), oscillations in the flow curve (2 patients, $2.6 \%$ ), and a biphasic expiratory curve (15 patients, $19.7 \%$ ) (24), which is also described in 8 of 11 patients in a separate small study with TBM (25). This biphasic flow-volume loop as characterized by a sudden and significant drop in flow on the expiratory limb followed by a plateau of very low flow for the rest of the expiration is thought to be the classic pattern of ECAC. A low maximum FEF was also described in $81.6 \%$ of cases (24). 

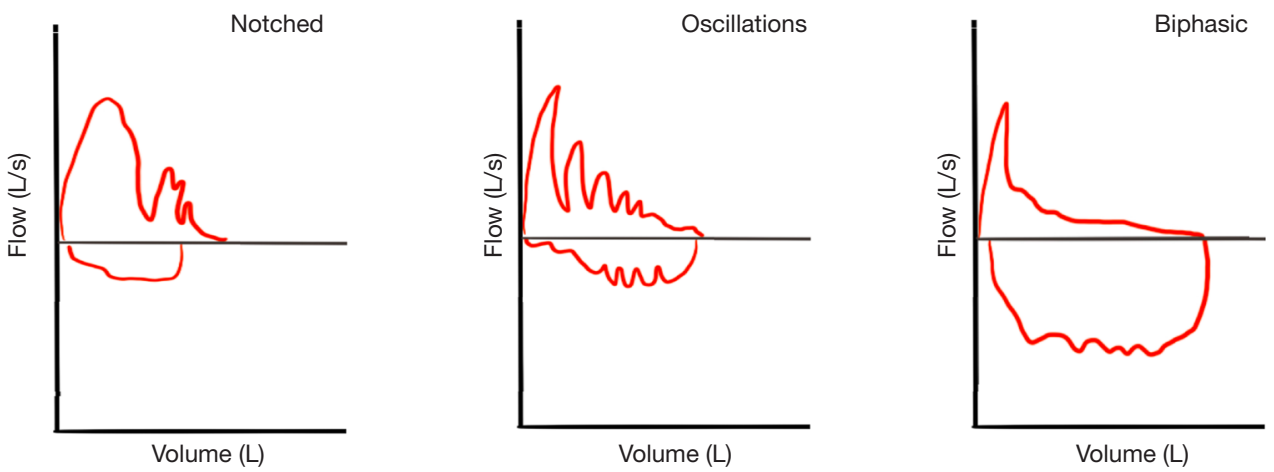

Figure 2 Representative models of flow-volume loops on PFT which may represent clinically important TBM. From left to right: notching of the expiratory curve, oscillations in the flow curve, and a biphasic expiratory limb of the curve. PFT, pulmonary function test; TBM, tracheobronchomalacia.

Given the significant variability of abnormal findings and patterns on spirometry, as well as the frequency of normal findings, routine PFT should not be used to confirm the presence of ECAC nor decide its clinical relevance when present. PFTs can be helpful in evaluating for other co-morbid lung conditions prior to any intervention for ECAC (3).

\section{Imaging}

Inspiratory and expiratory phase CT imaging can assist in classifying the morphological appearance of airway wall collapse. Frequently identified subtypes are saber sheath, circumferential, and crescent shaped. Both anterior cartilaginous wall weakness and posterior membranous collapse can be present in all morphologies and all are clinically significant. The degree of each can contribute to the distinct morphological appearance (3). In addition to morphology, a diagnosis of TBM is made when a reduction in cross-sectional area of the airway greater than or equal to $50 \%$ is identified on dynamic expiration (1).

\section{Multi-detector computed tomography}

Multiple different cross-sectional imaging techniques may be used for the diagnosis and evaluation of TBM, the most common of which is multi-detector computed tomography (MDCT). The three techniques of MDCT evaluation are: paired end inspiratory and end expiratory, paired end inspiratory and dynamic expiratory, and cine combined with a coughing maneuver (26-28). Intravenous contrast is not required for the routine assessment of ECAC (26).
However, it can be helpful in certain situations including known or suspected mediastinal vascular anomalies, compressive mediastinal masses, or central airway neoplasms (29).

With the paired end inspiratory and expiratory imaging technique, images are obtained at the end of inspiration and expiration. However, the end expiration technique is the least sensitive method for eliciting expiratory collapse, it is utilized for children $<5$ years of age who may not cooperate with dynamic expiratory breathing techniques $(26,30)$. With paired end inspiratory and dynamic expiratory imaging, images are obtained at end inspiration and during forced exhalation (dynamic expiration). This method is preferred for imaging adults and older children because tracheal collapse is best illustrated (Figure 3). Furthermore, this technique can be performed on any MDCT scanner though the best results are obtained on scanners with 8 or greater detector rows $(26,27)$. Before helical scanning, a standard topographic image is obtained to determine the scanning area approximating the proximal trachea to the mainstream bronchi, measuring on average 10 to $12 \mathrm{~cm}$ in adults. Both end inspiratory and dynamic expiratory scans are performed in the craniocaudal directions. The protocol involves initially obtaining the end inspiratory scan, then the patient is asked to take a deep breath in and blow it out forcefully during the acquisition of the expiratory scan (26). The last MDCT method is cine CT during coughing maneuvers. This technique requires use of a 64row or greater MDCT scanner. An initial topographic scout is obtained to determine the area of coverage with margins extending from the level of the aortic arch to the carina. Images are acquired in cine mode beginning at end 

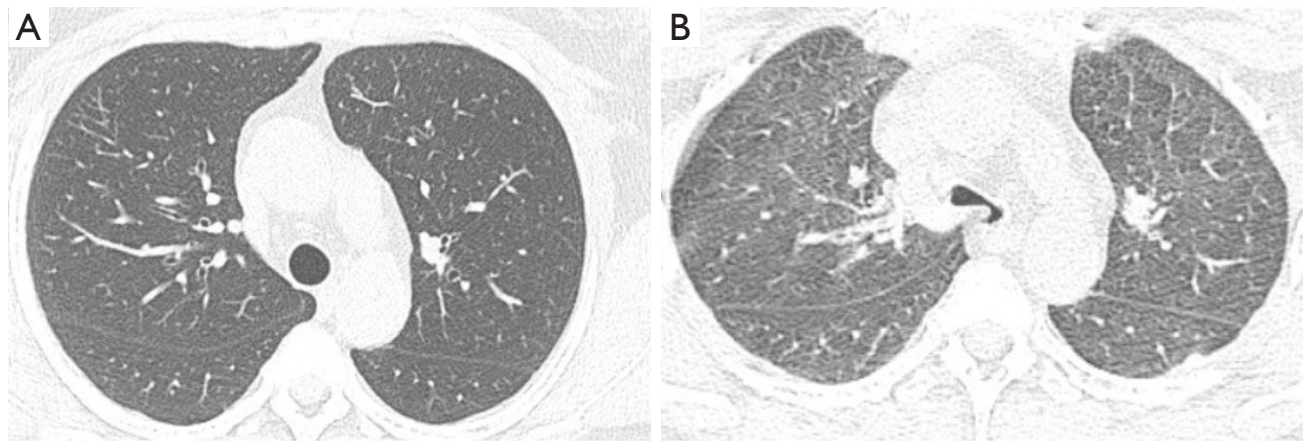

Figure 3 Axial nonenhanced CT images demonstrate normal tracheal lumen caliber on initial inspiratory image (A) with subsequent greater than $70 \%$ luminal decrease on dynamic expiration (B) consistent with severe tracheobronchomalacia. Note motion artifact on the dynamic images.
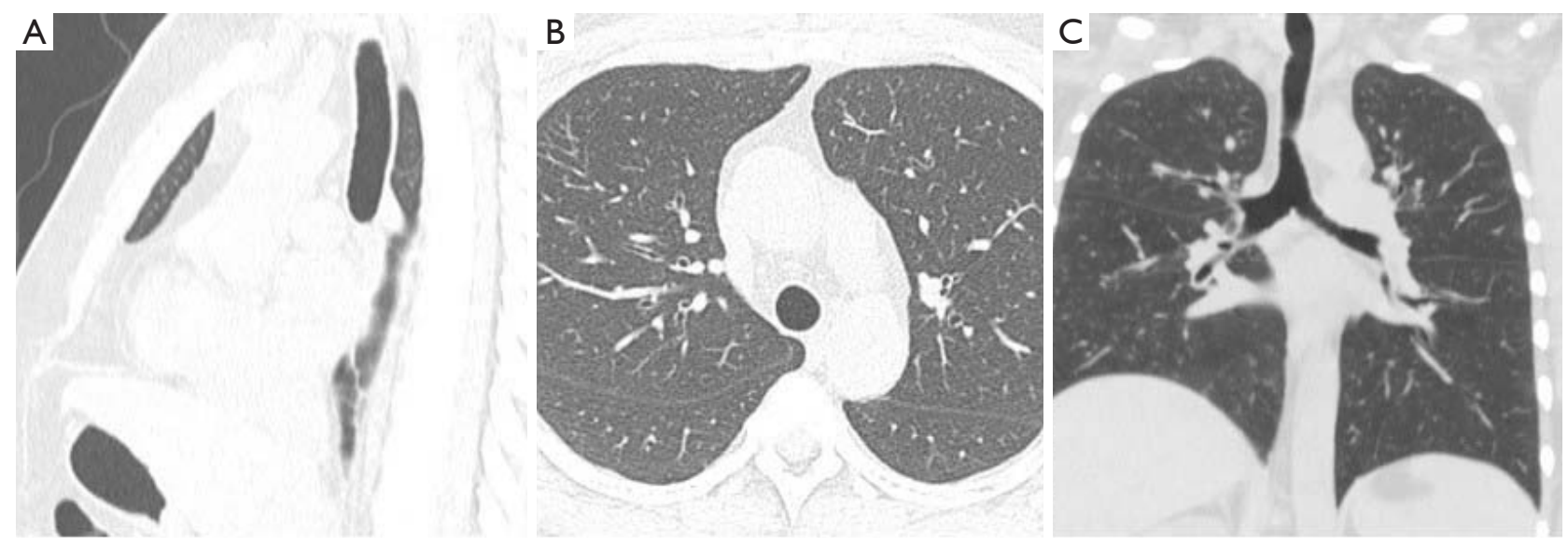

Figure 4 Multiplanar reconstruction of the trachea/central airways include sagittal (A), axial (B) and coronal (C) images. Trachea is normal in caliber.

inspiration and followed by repeating coughing maneuvers for approximately 5 secs and then reconstructed $(26,28)$.

\section{Consideration of dose}

Given CT imaging of ECAC has the potential to result in double the standard dose of a single-phase CT scan, methods of dose reduction are imperative to upholding the ALARA principle (as low as reasonably achievable) while maintaining diagnostic quality $(31,32)$. In fact, because of the inherent contrast between the air-filled trachea and surrounding soft tissue structures, it is possible to reduce dose without negatively affecting image quality. Dose modulation strategies include decreasing $\mathrm{mAs}$ and decreasing pitch (32). For instance, Zhang and colleagues demonstrated no difference between standard dose (240-
$280 \mathrm{~mA}$ ) and low dose (40-80 $\mathrm{mA})$ images for assessing tracheal lumen during dynamic expiration (31). Thus, a low dose technique should be utilized when conducting coughing or dynamic expiration images (31). Typically, the end inspiration scan is standard dose to assess for additional thoracic abnormalities, but dose modulation can also be used to further decrease radiation exposure $(31,32)$.

\section{Image quality/reconstruction}

To obtain a technically high-quality study, a thorough quality assurance process needs to be in place relying on adequate training of technologists to be able to coach and monitor patients as they perform the dynamic breathing techniques $(26,33)$.

Multi-planar reconstruction images can be created along 
any selected plane such as coronal, sagittal, orthogonal, or curved oblique planes (Figure 4). For airway imaging, curved oblique reformations are particularly useful for tubular structures such as the trachea and bronchi. Additional 3D volume rendering can be performed where 3D images are created following a threshold of Hounsfield attenuation using all the CT information initially acquired. $3 \mathrm{D}$ post processing techniques allow the image data to be manipulated for viewing from either the external or internal airways such as in CT bronchoscopy or virtual bronchoscopy methods $(26,34,35)$.

\section{Bronchoscopic evaluation}

Bronchoscopy is extremely helpful to evaluate the distribution, extent, and magnitude of collapse during dynamic breathing. It is performed under conscious sedation to allow patient cooperation. The Beth Israel group have published their protocol for bronchoscopic evaluation of ECAC $(11,20)$. The following is the protocol we use which is based on the BI protocol.

\section{Sedation/topical anesthesia}

We usually topically anesthetize the upper airway with $4 \%$ lidocaine. Once the gag reflex is sufficiently attenuated we provide sedation with midazolam and Fentanyl (typically $2-4 \mathrm{mg}$ midazolam and 50-100 mcg fentanyl). Additional 2\% lidocaine is delivered in $2-\mathrm{mL}$ aliquots to the vocal cords and then $1 \%$ lidocaine is delivered to the entire tracheobronchial tree.

\section{Positioning}

Typically, we position the patient in a supine recumbent position. We will start with 30-degree head elevation and assess the degree of airway collapse, occasionally if collapse is not pronounced, we will place the patient fully recumbent.

\section{Equipment}

An Olympus BF-Q190 video bronchoscope (Olympus America, Melville, NY, USA) with a 4.8-mm outer diameter and $2.0-\mathrm{mm}$ working channel or similar is used to minimize any possible stenting effect. Smaller bronchoscopes can be used but tend to be pushed around in the airway making image acquisition more difficult.

\section{Areas assessed}

Six areas are evaluated both during inspiration, forced expiration and coughing.

(I) LMB;

(II) Bronchus intermedius;

(III) Right main bronchus;

(IV) Distal trachea;

(V) Mid trachea;

(VI) Proximal trachea at the level of the thoracic inlet.

\section{Grading collapse}

The degree of collapse is assessed on forced expiration. We also assess the degree of collapse during coughing, we do this to make sure the forced expiratory maneuver was vigorous and there is not a large discrepancy between forced expiration and coughing. Elements to be evaluated during bronchoscopy include distribution, cartilaginous integrity and deformation shape, contraction of trachealis and secretions. We grade the degree of collapse as per the BIDMC grading:

(I) Mild 70-80\%;

(II) Moderate $80-90 \%$;

(III) Severe $>90 \%$.

Several other grading systems have been described but none universally accepted $(12,36)$. Majid $e t$ al. assessed the validity of a bronchoscopy protocol in the evaluation of TBM. They demonstrated that a bronchoscopic evaluation of airway collapse using still images at five different sites in the tracheobronchial tree had a moderate to strong interobserver agreement at baseline and intraobserver agreement 8 days after (evaluated by 23 pulmonologists not involved in the care of the study patients). This may be improved using video imaging (since airway collapse is a dynamic process) but provides supportive evidence for bronchoscopy as reliable diagnostic tool for ECAC (11). Shortcomings of our study include reliance on still rather than video images that are less susceptible to distortions and have the potential for greater accuracy because changes with TBM are dynamic.

\section{Summary}

Expiratory central airway collapse is an under recognized condition with significant symptom burden and attributable morbidity. It is commonly associated with chronic lung disease and obesity. Differentiating what component of a patient's symptoms can be attributed to ECAC is difficult. 
Modern imaging techniques have the ability to non-invasively diagnose ECAC and assess morphology; presently multidetector CT is the imaging modality of choice. Currently, we do not have a PFT to diagnose, grade severity of ECAC or predict response to surgical repair. Bronchoscopy remains the gold standard for diagnosis and functional assessment. We recommend multidisciplinary evaluation by a team familiar with the assessment and management of this condition prioritizing optimization of medical management before surgical intervention.

\section{Acknowledgments}

We would like to acknowledge Dr. Fusun Dikengil for her assistance in creating the flow-volume loop figure.

Funding: None.

\section{Footnote}

Provenance and Peer Review: This article was commissioned by the Guest Editors (Charles T. Bakhos and Abbas E. Abbas) for the series "Tracheobronchoplasty" published in Fournal of Visualized Surgery. The article has undergone external peer review.

Reporting Checklist: The authors have completed the Narrative Review reporting checklist. Available at https:// jovs.amegroups.com/article/view/10.21037/jovs-21-6/rc

Peer Review File: Available at https://jovs.amegroups.com/ article/view/10.21037/jovs-21-6/prf

Conflicts of Interest: All authors have completed the ICMJE uniform disclosure form (available at https://jovs. amegroups.com/article/view/10.21037/jovs-21-6/coif). The series "Tracheobronchoplasty" was commissioned by the editorial office without any funding or sponsorship. The authors have no other conflicts of interest to declare.

Ethical Statement: The authors are accountable for all aspects of the work in ensuring that questions related to the accuracy or integrity of any part of the work are appropriately investigated and resolved.

Open Access Statement: This is an Open Access article distributed in accordance with the Creative Commons Attribution-NonCommercial-NoDerivs 4.0 International License (CC BY-NC-ND 4.0), which permits the non- commercial replication and distribution of the article with the strict proviso that no changes or edits are made and the original work is properly cited (including links to both the formal publication through the relevant DOI and the license). See: https://creativecommons.org/licenses/by-nc-nd/4.0/.

\section{References}

1. Gilkeson RC, Ciancibello LM, Hejal RB, et al. Tracheobronchomalacia: dynamic airway evaluation with multidetector CT. AJR Am J Roentgenol 2001;176:205-10.

2. Boiselle PM, O'Donnell CR, Bankier AA, et al. Tracheal collapsibility in healthy volunteers during forced expiration: assessment with multidetector CT. Radiology 2009;252:255-62.

3. Buitrago DH, Wilson JL, Parikh M, et al. Current concepts in severe adult tracheobronchomalacia: evaluation and treatment. J Thorac Dis 2017;9:E57-66.

4. Weinstein DJ, Hull JE, Ritchie BL, et al. Exerciseassociated Excessive Dynamic Airway Collapse in Military Personnel. Ann Am Thorac Soc 2016;13:1476-82.

5. Castellanos P, Mk M, Atallah I. Laser tracheobronchoplasty: a novel technique for the treatment of symptomatic tracheobronchomalacia. Eur Arch Otorhinolaryngol 2017;274:1601-7.

6. Handa H, Miyazawa T, Murgu SD, et al. Novel multimodality imaging and physiologic assessments clarify choke-point physiology and airway wall structure in expiratory central airway collapse. Respir Care 2012;57:634-41.

7. Lee KS, Ernst A, Trentham DE, et al. Relapsing polychondritis: prevalence of expiratory CT airway abnormalities. Radiology 2006;240:565-73.

8. Macklem PT, Mead J. Factors determining maximum expiratory flow in dogs. J Appl Physiol 1968;25:159-69.

9. Smaldone GC, Smith PL. Location of flow-limiting segments via airway catheters near residual volume in humans. J Appl Physiol (1985) 1985;59:502-8.

10. MacKlem PT, Fraser RG, Brown WG. The detection of the flow-limiting bronchi in bronchitis and emphysema by airway pressure measurements. Med Thorac 1965;22:220-30.

11. Majid A, Gaurav K, Sanchez JM, et al. Evaluation of tracheobronchomalacia by dynamic flexible bronchoscopy. A pilot study. Ann Am Thorac Soc 2014;11:951-5.

12. Murgu S, Colt H. Tracheobronchomalacia and excessive dynamic airway collapse. Clin Chest Med 2013;34:527-55.

13. Boiselle PM, Michaud G, Roberts DH, et al. Dynamic 
expiratory tracheal collapse in COPD: correlation with clinical and physiologic parameters. Chest 2012;142:1539-44.

14. Ikeda S, Hanawa T, Konishi T, et al. Diagnosis, incidence, clinicopathology and surgical treatment of acquired tracheobronchomalacia. Nihon Kyobu Shikkan Gakkai Zasshi 1992;30:1028-35.

15. Jokinen K, Palva T, Nuutinen J. Chronic bronchitis. A bronchologic evaluation. ORL J Otorhinolaryngol Relat Spec 1976;38:178-86.

16. Gibson P, Wang G, McGarvey L, et al. Treatment of Unexplained Chronic Cough: CHEST Guideline and Expert Panel Report. Chest 2016;149:27-44.

17. Rotsides JM, Krakovsky GM, Pillai DK, et al. Is a Multidisciplinary Aerodigestive Clinic More Effective at Treating Recalcitrant Aerodigestive Complaints Than a Single Specialist? Ann Otol Rhinol Laryngol 2017;126:537-43.

18. Vertigan AE, Theodoros DG, Gibson PG, et al. Efficacy of speech pathology management for chronic cough: a randomised placebo controlled trial of treatment efficacy. Thorax 2006;61:1065-9.

19. Nygaard M, Hilberg O, Rasmussen F, et al. Tracheal collapsibility in adults is dynamic over time. Respir Med 2019;146:124-8.

20. Majid A, Kheir F, Alape D, et al. The Prevalence of Gastroesophageal Reflux in Patients With Excessive Central Airway Collapse. Chest 2019;155:540-5.

21. Bhatawadekar SA, Peters U, Walsh RR, et al. Central airway collapse is related to obesity independent of asthma phenotype. Respirology 2021;26:334-41.

22. Choo EM, Seaman JC, Musani AI. Tracheomalacia/ Tracheobronchomalacia and hyperdynamic airway collapse. Immunol Allergy Clin North Am 2013;33:23-34.

23. Kandaswamy C, Bird G, Gill N, et al. Severe tracheomalacia in the ICU: identification of diagnostic criteria and risk factor analysis from a case control study. Respir Care 2013;58:340-7.

24. Majid A, Sosa AF, Ernst A, et al. Pulmonary function and flow-volume loop patterns in patients with tracheobronchomalacia. Respir Care 2013;58:1521-6.

25. Campbell AH, Faulks LW. Expiratory air-flow pattern in tracheobronchial collapse. Am Rev Respir Dis 1965;92:781-91.

26. Lee EY, Litmanovich D, Boiselle PM. Multidetector CT evaluation of tracheobronchomalacia. Radiol Clin North
Am 2009;47:261-9.

27. Heussel CP, Hafner B, Lill J, et al. Paired inspiratory/ expiratory spiral CT and continuous respiration cine CT in the diagnosis of tracheal instability. Eur Radiol 2001;11:982-9.

28. Boiselle PM, Lee KS, Lin S, et al. Cine CT during coughing for assessment of tracheomalacia: preliminary experience with 64-MDCT. AJR Am J Roentgenol 2006;187:W175-7.

29. Chan MS, Chu WC, Cheung KL, et al. Angiography and dynamic airway evaluation with MDCT in the diagnosis of double aortic arch associated with tracheomalacia. AJR Am J Roentgenol 2005;185:1248-51.

30. Lee EY, Boiselle PM. Tracheobronchomalacia in infants and children: multidetector CT evaluation. Radiology 2009;252:7-22.

31. Zhang J, Hasegawa I, Feller-Kopman D, et al. 2003 AUR Memorial Award. Dynamic expiratory volumetric CT imaging of the central airways: comparison of standard-dose and low-dose techniques. Acad Radiol 2003;10:719-24.

32. Choi YW, McAdams HP, Jeon SC, et al. Low-dose spiral CT: application to surface-rendered three-dimensional imaging of central airways. J Comput Assist Tomogr 2002;26:335-41.

33. Bankier AA, O'Donnell CR, Boiselle PM. Quality initiatives. Respiratory instructions for CT examinations of the lungs: a hands-on guide. Radiographics 2008;28:919-31.

34. Boiselle PM. Tracheomalacia: functional imaging of the large airways with multidetector-row CT. In: Boiselle PM, White CS. editors. New techniques in cardiothoracic imaging. New York: Informa, 2007:177-85.

35. Calhoun PS, Kuszyk BS, Heath DG, et al. Threedimensional volume rendering of spiral CT data: theory and method. Radiographics 1999;19:745-64.

36. Murgu SD, Colt HG. Description of a multidimensional classification system for patients with expiratory central airway collapse. Respirology 2007;12:543-50.

doi: 10.21037/jovs-21-6

Cite this article as: Ramakrishnan K, Kumaran M, Townsend R, Weir M. Clinical advances in the evaluation of patients with excessive central airway collapse: a narrative review. J Vis Surg 2022;8:13. 